\title{
Custos, Desempenho e Estrutura Educacional do Ensino Fundamental: Uma Análise em um Município do Interior do Paraná
}

\begin{abstract}
Lealis Vaz Meleiro Lopes
Mestrado em andamento em Administração pela Universidade de São Paulo - USP

E-mail: lealisvaz@gmail.com

Caroline Nayara Marilac Flor Graduação em andamento em Administração pela Universidade Estadual de Londrina UEL E-mail: carolinemarilacf@gmail.com

Marissa Yanara de Godoy Lima Mestrado em Administração pela Universidade Estadual de Londrina - UEL Agente Local de Inovação no Serviço de Apoio às Micro e Pequenas Empresas -

SEBRAE

E-mail: marissa-godoy@uol.com.br

Saulo Fabiano Amâncio Vieira Doutorado em Administração pela Universidade Nove de Julho - UNINOVE Professor da Universidade Estadual de Londrina - UEL Rod. Celso Garcia Cid, s/n. Campus Universitário. Londrina/PR. Brasil. CEP: 86.057-

E-mail: saulofav@gmail.com

Thais Accioly Baccaro Doutorado em Administração pela Faculdade de Economia, Administração e Contabilidade de Ribeirão Preto - FEARP/USP Professora Adjunta da Universidade Estadual de Londrina - UEL Rod. Celso Garcia Cid, s/n. Campus Universitário. Londrina/PR. Brasil. CEP: 86.057-

E-mail: thaisbaccaro@uel.br
\end{abstract}

\section{RESUMO}

Medidas eficientes por parte dos gestores públicos são necessárias para que os recursos destinados à educação sejam distribuídos de maneira igualitária e justa a toda a sociedade. Diante dessa afirmação, nota-se a necessidade de mensurar adequadamente o custo do sistema educacional, garantindo que os gastos públicos destinados ao desenvolvimento educacional sejam empregados tão somente para essa finalidade. Perante a situação de desigualdade no ensino, este artigo tem por objetivo analisar se as escolas de Santa Terezinha de Itaipu/PR, que possuem o maior custo 
Custos, Desempenho e Estrutura Educacional do Ensino Fundamental: Uma Análise em um Município do Interior do Paraná Lealis Vaz Meleiro Lopes, Caroline Nayara Marilac Flor, Marissa Yanara de Godoy Lima, Saulo Fabiano Amâncio Vieira, Thais Accioly Baccaro

por aluno, são também aquelas que ostentam o melhor desempenho no IDEB, identificando outros possíveis fatores que venham a influenciar sua performance. $O$ estudo analisa, de forma quantitativa e descritiva, a distribuição dos recursos investidos na educação do Ensino Fundamental em um município de pequeno porte, localizado no interior do Paraná. Verificou-se que as escolas que apresentaram maior custo/aluno não necessariamente possuem o melhor desempenho nos indicadores de qualidade do Índice de Desenvolvimento da Educação Básica (IDEB). Contudo, a relação custo/aluno entre as escolas é relativamente simétrica, havendo uma diferença de apenas $\mathrm{R} \$ 39,00$ entre a escola com maior custo daquela com menor custo, o que representa $24,62 \%$ de discrepância em relação ao custo médio.

Palavras-chave: Custo no Setor Público. Gestão Pública. Desempenho. Ensino Fundamental.

\section{Costs, Performance and Educational Structure of Elementary School: An Analysis in a Municipality of the Interior of Paraná}

\section{ABSTRACT}

Efficient measures by public managers are necessary so that the re-courses for education are distributed equally and fairly to all society. In view of this assertion, it is necessary to measure the cost of the educational system adequately, ensuring that public expenditures for educational development are used only for this purpose. In view of the situation of inequality in education, this article aims to analyze if the schools of Santa Terezinha de Itaipu / PR that have the highest cost per student are also those that have the best performance in the IDEB, identifying other possible factors that come to influence their performance. The study quantitatively and descriptively analyzes the distribution of resources invested in elementary school education in a small municipality located in the interior of Paraná. It was verified that the schools that presented the highest cost / student did not necessarily present better performance in the indicators of quality of the Basic Education Development Index (IDEB). However, the cost / student ratio between schools is relatively symmetrical, with a difference of only $R \$ 39,00$ between the school with the highest cost of the one with the lowest cost, totaling a $24.62 \%$ discrepancy in relation to the average cost.

Key-words: Cost in the Public Sector. Public administration. Performance. Elementary School. 
Custos, Desempenho e Estrutura Educacional do Ensino Fundamental: Uma Análise em um Município do Interior do Paraná Lealis Vaz Meleiro Lopes, Caroline Nayara Marilac Flor, Marissa Yanara de Godoy Lima, Saulo Fabiano Amâncio Vieira, Thais Accioly Baccaro

\section{INTRODUÇÃO}

Um diagnóstico preciso da situação do Ensino Fundamental no Brasil tem como finalidade verificar se os direitos constitucionais, garantidos pela Constituição Federal de 1988 (Brasil, 1988), sobretudo no que diz respeito à educação, estão sendo cumpridos. De acordo com a legislação (Brasil, 1988), a educação é direito de todos e dever do Estado. A Constituição Federal declara como princípios do ensino não somente a igualdade de condições de acesso e permanência, mas a correspondente obrigação de oferta de uma escola com um padrão de qualidade que possibilite a todos os brasileiros cursar uma escola com boas condições de funcionamento e de competência educacional, em termos de pessoal, material, recursos financeiros e projeto pedagógico que Ihes permita identificar e reivindicar a "escola de qualidade comum" de direito de todos os cidadãos (Arelaro, 2005).

O Índice de Desenvolvimento da Educação Básica [IDEB] aponta que alunos do Ensino Fundamental apresentam baixo desempenho nesta avaliação (INEP, 2016). Ao averiguar como são escassos os recursos em prol do ensino no Brasil, há uma comparação com o ensino dos países mais desenvolvidos, que denotam grande discrepância em questão de qualidade e evolução do sistema educacional.

As fontes que disponibilizam informações de gastos públicos são muito escassas, dificultando os procedimentos para uma gestão eficaz. A preocupação aumenta à medida que os dados disponíveis apontam para um baixo retorno em relação aos gastos públicos destinados à educação. Assim, o presente artigo busca responder a seguinte questão: as escolas de Santa Terezinha de Itaipu/PR que possuem o maior custo por aluno são também aquelas que ostentam o melhor desempenho no Índice de Desenvolvimento da Educação Básica (IDEB)?

O estudo realizou-se a partir de uma coleta de dados efetuada por meio do portal IDEB e NSE (Nível Socioeconômico), visando mensurar e analisar os gastos públicos referentes à educação básica do Ensino Fundamental. 
Custos, Desempenho e Estrutura Educacional do Ensino Fundamental: Uma Análise em um Município do Interior do Paraná Lealis Vaz Meleiro Lopes, Caroline Nayara Marilac Flor, Marissa Yanara de Godoy Lima, Saulo Fabiano Amâncio Vieira, Thais Accioly Baccaro

Este estudo justifica-se por reforçar a importância de se estudar os custos relativos ao setor público, especialmente no que se refere à educação. Assim, visa contribuir, de forma teórica e empírica, para a temática abordada, oferecendo subsídios para o melhoramento da gestão pública municipal.

O presente artigo divide-se em quatro partes: a primeira é referente à introdução, a segunda ao referencial teórico com base na situação educacional brasileira e no IDEB; em seguida, são apresentados os procedimentos metodológicos e, na sequência, a análise dos resultados e as considerações finais.

\section{REFERENCIAL TEÓRICO}

O referencial teórico disserta sobre a educação enquanto conceito e descreve um breve histórico da educação brasileira, abordando o IDEB como indicador de qualidade do Ensino Fundamental e a Gestão de Custos como instrumento de controle.

\subsection{Educação}

Garantido pela Constituição Federal (Brasil, 1988), o acesso à educação é direito de todos e está disponível como um serviço público, desde que prestado pelo Estado. Trata-se de um processo formativo presente em praticamente todas as etapas da vida, não sendo exclusividade da vida estudantil, mas desenvolvida de maneira mais significativa durante esta fase.

O Brasil apresenta, de forma agravada, algumas características próprias de países em desenvolvimento, entre as quais: enorme desigualdade na distribuição da renda e consideráveis deficiências no sistema educacional (Goldemberg, 1993).

Esses dois problemas estão diretamente associados. Não é possível aumentar a renda média de adultos sem instrução nem educar adequadamente crianças cujas famílias vivem à beira da miséria. Por este motivo, ao se traçar uma política educacional, há de se evitar a posição simplista de que se pode resolver o problema da pobreza apenas abrindo escolas. Pobreza e ausência de escolarização são 
Custos, Desempenho e Estrutura Educacional do Ensino Fundamental: Uma Análise em um Município do Interior do Paraná Lealis Vaz Meleiro Lopes, Caroline Nayara Marilac Flor, Marissa Yanara de Godoy Lima, Saulo Fabiano Amâncio Vieira, Thais Accioly Baccaro

deficiências que somente poderão ser superadas se enfrentadas simultaneamente, cada uma em seu lugar próprio (Goldemberg, 1993). Mediante esse contexto, o autor afirma que:

Apesar do muito já conseguido, as deficiências do sistema educacional brasileiro constituem certamente um entrave para a modernização da sociedade e é necessário reconhecer que: a qualidade do ensino, tanto público como privado e em todos os níveis é, na maioria dos estabelecimentos, muito deficiente; apesar da quase universalização do acesso à escola que se logrou atingir, uma porcentagem demasiado pequena dos alunos consegue completar os oito anos de ensino básico obrigatório; o ensino de nível médio não consegue preparar adequadamente os estudantes para a universidade, nem para o ingresso no mundo do trabalho; a formação oferecida nas universidades não promove a qualificação que seria necessária; e a pesquisa é, no mais das vezes, incipiente ou inexistente (Goldemberg, 1993, p. 70).

Diante disso, Dourado (2007) coloca que as políticas educacionais no Brasil têm sido marcadas hegemonicamente pela lógica da descontinuidade, por falta de planejamento em longo prazo que evidenciasse uma política de Estado, e não simplesmente uma política conjuntural de governo. Para o autor, tal dinâmica favorece ações sem articulação suficiente com os sistemas de ensino, que é observada pela gestão e organização insuficientes, pela falta de formação inicial e continuada, pela desatualização da estrutura curricular e dos processos de participação. Em um resgate histórico, o autor discorre que:

[...] desde a redemocratização do país, houveram mudanças acentuadas na educação brasileira, com destaque para a aprovação e promulgação da Constituição Federal de 1988, que garantiu uma concepção ampla de educação e sua inscrição como direito social inalienável, bem como a partilha de responsabilidade entre os entes federados e a vinculação constitucional de recursos para os de gestão no sistema educacional que os conceitos da lógica capitalista de produtividade e racionalidade fossem inclusos na esfera pública. Em tal período, se instaurou uma gestão centralizada das políticas educacionais, e não resolveram a realidade educacional excludente e seletiva vigente que permanece até a atualidade (Dourado, 2007, p. 926).

A partir de então, observa-se que a gestão centralizada das políticas educacionais se torna ineficaz diante das atuais demandas, legitimando a baixa qualidade do ensino no país. 
Custos, Desempenho e Estrutura Educacional do Ensino Fundamental: Uma Análise em um Município do Interior do Paraná Lealis Vaz Meleiro Lopes, Caroline Nayara Marilac Flor, Marissa Yanara de Godoy Lima, Saulo Fabiano Amâncio Vieira, Thais Accioly Baccaro

É necessário salientar, porém, que evoluções na educação brasileira são possíveis e já estão ocorrendo. Dados do IBGE (2018) apontam que a taxa de analfabetismo de pessoas entre 10 e 14 anos, em 2007, era de 3,1\% e que, em 2015, passou a ser de $1,6 \%$. Para pessoas acima de 15 anos, a taxa de analfabetismo era de 10,1\% em 2007 e passou a ser de 8\% em 2015. A taxa de escolarização de pessoas entre 6 e 14 anos se manteve estável. Em 2007, 97\% delas estavam na escola e, em 2015, esse índice subiu para 98,6\%.

O ensino de qualidade torna-se ainda mais urgente na contemporaneidade, já que a sociedade atual é, por vezes, denominada como "sociedade do conhecimento, sociedade técnico-informacional ou sociedade tecnológica, o que significa que 0 conhecimento, o saber e a ciência assumem um papel muito mais destacado que anteriormente" (De Oliveira, Libâneo \& Toschi, 2017). Desse modo, a escola sofre pressão não só para que o ensino seja melhor, mas também para se integrar a modalidades de ensino informal a fim de "formar cidadãos mais qualificados e preparados para um novo tempo" (De Oliveira, et al. 2017). Nesse sentido, têm-se alguns indicadores educacionais que visam mensurar o desempenho das unidades escolares e apoiar as ações do Estado.

\subsection{Indicadores Educacionais}

Para medir a qualidade de serviços oferecidos à sociedade, a presente pesquisa utiliza os dados de custos educacionais coletados por meio do Sistema Integrado de Custos Municipais (SICm), IDEB e Nível Socioeconômico (NSE), que serão detalhados a seguir.

O SICm foi desenvolvido pela Universidade Estadual de Londrina (UEL), em convênio com a Secretaria da Ciência, Tecnologia e Ensino Superior (SETI), e tem por objetivo auxiliar os gestores públicos no processo de tomada de decisão. Esse sistema calcula os custos das unidades escolares municipais e contribui para o planejamento, execução e avaliação da gestão educacional do município (Sistema Integrado de Custos Municipais, 2015). 
Custos, Desempenho e Estrutura Educacional do Ensino Fundamental: Uma Análise em um Município do Interior do Paraná Lealis Vaz Meleiro Lopes, Caroline Nayara Marilac Flor, Marissa Yanara de Godoy Lima, Saulo Fabiano Amâncio Vieira, Thais Accioly Baccaro

O IDEB é um indicador de qualidade utilizado para averiguar o desempenho escolar dos alunos de uma instituição (INEP, 2015). Por meio deste, é possível que o governo constate se as mudanças propostas no ensino escolar atingiram os objetivos propostos.

Soares e Pereira (2013) explicam que o desempenho de uma escola é definido para o cálculo do IDEB como a média das proficiências em Leitura e Matemática obtidas pelos seus alunos na Prova Brasil. Como as escalas das medidas das duas competências são diferentes, faz-se uma padronização dessas medidas antes de se calcular sua média. A metodologia do IDEB assume que, para tornar comparáveis as proficiências de Leitura e Matemática, basta fazer com que variem no mesmo intervalo (INEP, 2015). Exemplo disso é a fixação de uma nota média a ser atingida pelos estudantes até 2021. Essa meta foi estabelecida pelo Plano de Educação (PDE), eixo do Ministério da Educação, responsável pela Educação Básica, e visa atingir a nota 6,0. De acordo com a Organização para Cooperação e Desenvolvimento Econômico, cumprir essa meta significa se aproximar da média dos países desenvolvidos (Fernandes, 2007).

O nível socioeconômico (NSE) é definido pelo Portal QEdu (2016) como um indicador que "sintetiza as características dos indivíduos em relação à sua renda, ocupação e escolaridade". Tal indicador é utilizado para fazer análises de indivíduos semelhantes de acordo com estas características. É um valor numérico, em uma escala que varia de 0 a 10, e pode ser classificada como "mais baixo", "baixo", "médio baixo", "médio", "médio alto", "alto" e "mais alto". Esse indicador justifica-se por uma possível relação entre os resultados escolares e o nível socioeconômico e cultural das famílias. Quanto mais alto o NSE, maior a probabilidade de se obter uma maior escolaridade e acesso educacional.

Segundo as quatro edições já divulgadas pelo Instituto Nacional de Estudos e Pesquisas Educacionais Anísio Teixeira (INEP), as notas das escolas do Ensino Fundamental de Santa Terezinha do Itaipu referentes aos anos 2007, 2009, 2011 e 
Custos, Desempenho e Estrutura Educacional do Ensino Fundamental: Uma Análise em um Município do Interior do Paraná Lealis Vaz Meleiro Lopes, Caroline Nayara Marilac Flor, Marissa Yanara de Godoy Lima, Saulo Fabiano

Amâncio Vieira, Thais Accioly Baccaro

2013 foram, respectivamente, 5,2; 6,4; 7,5 e 6,5, demonstrando uma possível melhoria e, em seguida, um declínio (INEP, 2016).

Com base no desempenho das escolas, é necessário averiguar, nas próximas avaliações, se haverá evolução ou declínio no indicador, para então afirmar se as metas pré-estabelecidas pelo PDE podem ser cumpridas dentro do prazo.

\subsection{Gestão de Custos na Administração Pública}

Nas últimas décadas, o modelo de gestão pública vem evoluindo e buscando alcançar resultados mais eficazes com custos mais racionais. $O$ administrador público passou a deparar-se com o novo ambiente global, composto de novas exigências sociais, tecnologias e projetos, que tem originado, por sua vez, um novo modelo de gestão pública (Vendramin Junior, 2015).

Um estudo de Jackson e Lapsey (2003) aponta que existe uma tendência de haver progressivamente inovações contábeis no setor público. Novas técnicas, tais como cálculo de custos com base em atividades, gerenciamento de recursos e indicadores-chave de desempenho estão sendo introduzidas em muitas organizações do setor público. No entanto, as técnicas contábeis em uso no setor público são principalmente na recomendação de órgãos oficiais. Assim, quando a mudança contábil ocorre, acontece porque é forçada por regulação, estatuto ou exortação.

Para Martins (2009), por custo entende-se:

Gasto relativo a bem ou serviço utilizado na produção de outros bens e serviços. O custo é também um gasto, só que reconhecido como tal, isto é, como custo, no momento da utilização dos fatores de produção (bens e serviços), para a fabricação de um produto ou execução de um serviço (Martins, 2009).

Mediante uma conceituação prévia, o autor separa os custos em duas categorias: 
Custos, Desempenho e Estrutura Educacional do Ensino Fundamental: Uma Análise em um Município do Interior do Paraná Lealis Vaz Meleiro Lopes, Caroline Nayara Marilac Flor, Marissa Yanara de Godoy Lima, Saulo Fabiano Amâncio Vieira, Thais Accioly Baccaro

[...] podemos verificar que alguns custos podem ser diretamente apropriados aos produtos, bastando haver uma medida de consumo (quilogramas de materiais consumidos, embalagens utilizadas, horas de mão-de-obra utilizadas e até quantidade de força consumida). São os Custos Diretos com relação aos produtos. Outros realmente não oferecem condição de uma medida objetiva, e qualquer tentativa de alocação tem de ser feita de maneira estimada e muitas vezes arbitrária (como o aluguel, a supervisão, as chefias, etc.). São os Custos Indiretos com relação aos produtos (Martins, 2009, p. 32, grifo nosso).

Desse modo, para a presente pesquisa, serão considerados os custos diretos incidentes às unidades escolares, pois são os que interferem diretamente no ambiente escolar.

\subsection{O Município de Santa Terezinha de Itaipu}

De acordo com a Prefeitura Municipal (2016), Santa Terezinha de Itaipu é uma cidade do Oeste Paranaense, localizada a 610 quilômetros da capital (Curitiba) e a 28 quilômetros de Foz do Iguaçu. Possui área territorial de $267.491 \mathrm{~km}^{2}$, solo e clima favoráveis à produção de grãos, principalmente às culturas de soja, milho e trigo. $\mathrm{Na}$ rede hidrográfica de Santa Terezinha de Itaipu, os rios que se destacam são: Tamanduá, São João, Tucano, Bonito, Apepu, Guabirova, Gaviroba e Leão. Com exceção do Rio Apepu, todos têm nascente no município.

A população do município é de 20.834 pessoas, composta por $51,07 \%$ de mulheres e 48,93\% de homens (Portal População, 2016). De acordo com dados da Prefeitura Municipal de Santa Terezinha de Itaipu (2016), quase 34\% da população vive na zona rural.

Conforme a Prefeitura Municipal (2016), Santa Terezinha de Itaipu é um dos 15 municípios paranaenses que recebem Royalties da Itaipu Binacional. Até o dia 2 de maio de 1982, Santa Terezinha de Itaipu, que era chamada de Vila Criciúma, pertencia a Foz do Iguaçu. No dia 3 de maio de 1982, tornou-se independente. O nome é em homenagem à santa padroeira da cidade, Santa Terezinha. "Itaipu" é uma palavra de origem tupi-guarani, significando "barulho do rio da pedra" (Portal Cidades do Meu Brasil, 2016). 
Custos, Desempenho e Estrutura Educacional do Ensino Fundamental: Uma Análise em um Município do Interior do Paraná Lealis Vaz Meleiro Lopes, Caroline Nayara Marilac Flor, Marissa Yanara de Godoy Lima, Saulo Fabiano Amâncio Vieira, Thais Accioly Baccaro

\section{PROCEDIMENTOS METODOLÓGICOS}

A presente pesquisa tem perfil exploratório, descritivo, dispondo de abordagem quantitativa e é desenvolvida mediante estudo de caso. Trata-se, primeiramente, de um estudo exploratório, pois visa responder a um fenômeno cujas pesquisas são escassas e tende a procurar tendências, padrões ou segmentos; descritiva por se propor descrever dados e fatos pertinentes a um fenômeno; e quantitativa por consistir na comparação dos custos, estrutura e desempenho das escolas de Ensino Fundamental do município de Santa Terezinha do Itaipu.

A coleta de dados foi conduzida da seguinte forma: primeiramente, acessou-se o relatório referente ao estudo dos custos das escolas públicas de Santa Terezinha de Itaipu do ano de 2013, disponibilizado no site < https://sicm.dc.uel.br>. Posteriormente, foram feitas as comparações entre custo e desempenho das escolas públicas. Por fim, foi feito o georreferenciamento e um gráfico para ilustrar os resultados. Por meio de um censo, que considerou todas as unidades escolares municipais de Santa Terezinha do Itaipu, analisaram-se as cinco escolas públicas do município, focando nos anos iniciais do Ensino Fundamental, que compreende alunos de seis a dez anos e totaliza 1.794 alunos matriculados.

\section{RESULTADOS}

A apresentação dos resultados está dividida em: Descrição da Pesquisa, Análise de Custos e Análise de Desempenho.

\subsection{Descrição da Pesquisa}

Com relação ao número de alunos matriculados nas escolas do município de Santa Terezinha de Itaipu, encontraram-se os seguintes dados: 562 alunos (9,9\% dos alunos) são da educação infantil; 1.795 (31,46\%) são do Ensino Fundamental 1 (do $1^{\circ}$ 
Custos, Desempenho e Estrutura Educacional do Ensino Fundamental: Uma Análise em um Município do Interior do Paraná Lealis Vaz Meleiro Lopes, Caroline Nayara Marilac Flor, Marissa Yanara de Godoy Lima, Saulo Fabiano Amâncio Vieira, Thais Accioly Baccaro

ao $5^{\circ}$ ano); 1.491 alunos (26,14\% da população) são do Ensino Fundamental 2 (do $6^{\circ}$ ao $9^{\circ}$ ano), 434 alunos (24,9\% da população) são do EJA (Educação de Jovens e Adultos); e 1.420 (24,9\%) são outros alunos atendidos pelo município. Observa-se, de acordo com as informações obtidas, que a maior parte das matrículas ativas se refere ao Ensino Fundamental, o que representa $31,46 \%$ do número total de alunos.

Em relação ao número de alunos matriculados por escola, 242 foram matriculados na escola Alexandre Zilli Netto, 347 na escola Cecília Meireles, 336 na escola Monteiro Lobato, 416 na escola Nossa Senhora do Carmo e 337 na Olímpio Spricigo.

Nota-se, a partir das informações apresentadas, que a média de alunos por unidade escolar é de 336, em que o mínimo registrado é 242 (Alexandre Zilli Netto) e o máximo 416 (Nossa Senhora do Carmo).

\subsection{Análise de Custos}

A partir dos dados coletados no portal SICm (2016), observa-se pouca variação nos custos diretos das escolas de Santa Terezinha de Itaipu. Nota-se um custo mínimo de $\mathrm{R} \$ 131,00$ e máximo de $\mathrm{R} \$ 170,00$ para os alunos do Ensino Infantil. De acordo com o CAQi (Custo Aluno Qualidade inicial), para os primeiros anos do Ensino Fundamental, o valor do Custo Aluno nacional é de $R \$ 182,88$. Já no estado do Paraná, o Custo Aluno é de $\mathrm{R} \$ 178,09$; portanto, o município possui um Custo/Aluno menor que a média nacional.

Na presente pesquisa, os custos são divididos em Custos Pedagógicos, Custos Sociais e Custos Administrativos, conforme a Tabela 1. Os Custos Pedagógicos compreendem, entre outros valores, os custos com professores. Já nos Custos Sociais, estão os valores relativos à mão de obra da cozinha e gêneros alimentícios. Assim, para os Custos Administrativos, restam os gastos com técnicos e demais funcionários. 
Custos, Desempenho e Estrutura Educacional do Ensino Fundamental: Uma Análise em um Município do Interior do Paraná Lealis Vaz Meleiro Lopes, Caroline Nayara Marilac Flor, Marissa Yanara de Godoy Lima, Saulo Fabiano

Tabela 1

Unidades escolares e seus respectivos custos

\begin{tabular}{|c|c|c|c|c|}
\hline Escola & $\begin{array}{c}\text { Custo } \\
\text { Pedagógico }\end{array}$ & $\begin{array}{c}\text { Custo } \\
\text { Administrativo }\end{array}$ & Custo Social & Custo Total \\
\hline $\begin{array}{l}\text { ALEXANDRE ZILLI } \\
\text { NATTO E M E INF E } \\
\text { FUND }\end{array}$ & $R \$ 146,63$ & $\mathrm{R} \$ 13,90$ & $\mathrm{R} \$ 10,20$ & $R \$ 170,73$ \\
\hline $\begin{array}{l}\text { CECÍCLIA MEIRELES } \\
\text { E M ED INF ENS FUND }\end{array}$ & $\mathrm{R} \$ 139,54$ & $\mathrm{R} \$ 8,34$ & $\mathrm{R} \$ 11,64$ & $\mathrm{R} \$ 159,52$ \\
\hline $\begin{array}{l}\text { MONTEIRO LOBATO E } \\
\text { M ED INF ENS FUND }\end{array}$ & $\mathrm{R} \$ 129,06$ & $\mathrm{R} \$ 9,87$ & $\mathrm{R} \$ 12,84$ & $\mathrm{R} \$ 151,76$ \\
\hline $\begin{array}{l}\text { NOSSA SENHORA DO } \\
\text { CARRMO E M E INF E } \\
\text { FUND }\end{array}$ & $\mathrm{R} \$ 114,13$ & $\mathrm{R} \$ 7,19$ & R4 10,21 & $R \$ 131,52$ \\
\hline $\begin{array}{l}\text { OLÍMPIO SPRICIGO E } \\
\text { M ENS FUND }\end{array}$ & R\$ 163,34 & $\mathrm{R} \$ \mathbf{4 , 4 0}$ & $R \$ 14,08$ & $\mathrm{R} \$ 181,82$ \\
\hline
\end{tabular}

Nota. Fonte: Elaborada pelos autores.

Com um valor médio de $R \$ 159,07$, percebe-se que a maior parte dos custos educacionais refere-se ao custo pedagógico. Valores relativos aos custos administrativos e sociais não possuem muita representatividade, e sua soma corresponde a $15 \%$ do valor total. Diante dos valores apresentados, tem-se que as escolas municipais de Santa Terezinha do Itaipu possuem um baixo custo frente à média do estado e do país.

Mediante a exposição dos dados acima, foi gerada uma nova tabela, visando demonstrar a média do custo/aluno de cada uma das áreas (pedagógica, social e administrativa), bem como a representatividade de tais custos em relação ao custo total. 
Custos, Desempenho e Estrutura Educacional do Ensino Fundamental: Uma Análise em um Município do Interior do Paraná Lealis Vaz Meleiro Lopes, Caroline Nayara Marilac Flor, Marissa Yanara de Godoy Lima, Saulo Fabiano

Tabela 2

Média dos Custos Pedagógicos, Sociais e Administrativos

\begin{tabular}{|l|c|c|}
\hline \multicolumn{1}{|c|}{ Classificação } & $\begin{array}{c}\text { Média do } \\
\text { Custo/Aluno }\end{array}$ & Representatividade Média \\
\hline Custos Pedagógicos & $\mathrm{R} \$ 138,54$ & $\mathbf{8 7 , 0 9 \%}$ \\
\hline Custos Sociais & $\mathrm{R} \$ 11,79$ & $\mathbf{7 , 4 1 \%}$ \\
\hline Custos Administrativos & $\mathrm{R} \$ 8,74$ & $\mathbf{5 , 4 9 \%}$ \\
\hline Total & $\mathbf{1 5 9 , 0 7}$ & $\mathbf{1 0 0 \%}$ \\
\hline
\end{tabular}

Nota. Fonte: Elaborada pelos autores.

A partir da Tabela 2, utilizando-se o Custo Direto Médio, estimou-se a média dos custos pedagógicos, sociais e administrativos. Ratificou-se, portanto, que a maior parte dos valores calculados refere-se a Custos Pedagógicos $(87,09 \%)$, seguido dos Custos Sociais $(7,419 \%)$ e dos Custos Administrativos (5,49\%).

Tabela 3

Custo por aluno para o georreferenciamento

\begin{tabular}{|c|c|c|c|}
\hline Custo Aluno & $\begin{array}{c}\text { Quantidade de } \\
\text { Escolas }\end{array}$ & Cor no mapa & Representatividade \\
\hline $\mathbf{R} \$ \mathbf{1 3 0}$ a $\mathbf{R} \$ \mathbf{1 4 0}$ & 1 & Amarelo & $\mathbf{2 0 \%}$ \\
\hline $\mathbf{R} \mathbf{1 4 1}$ a $\mathbf{R} \mathbf{1 5 0}$ & 0 & Verde & $\mathbf{0}$ \\
\hline $\mathbf{R} \$ 151$ a $\mathbf{R} \mathbf{1 6 0}$ & 2 & Azul & $\mathbf{4 0 \%}$ \\
\hline $\mathbf{R} \mathbf{1 6 1}$ a $\mathbf{1 7 0}$ & 0 & Marrom & $\mathbf{0}$ \\
\hline Acima de $\mathbf{R} \$ \mathbf{1 7 0}$ & $\mathbf{2}$ & Vermelho & \\
\hline
\end{tabular}

Nota. Fonte: Elaborada pelos autores.

$\mathrm{Na}$ Tabela 3, encontram-se as faixas de valores relativas ao Custo Aluno e sua representatividade. Esta servirá de auxílio para localizar as unidades escolares no mapa geográfico de Santa Terezinha do Itaipu, que será apresentado a seguir: 
Custos, Desempenho e Estrutura Educacional do Ensino Fundamental: Uma Análise em um Município do Interior do Paraná Lealis Vaz Meleiro Lopes, Caroline Nayara Marilac Flor, Marissa Yanara de Godoy Lima, Saulo Fabiano Amâncio Vieira, Thais Accioly Baccaro

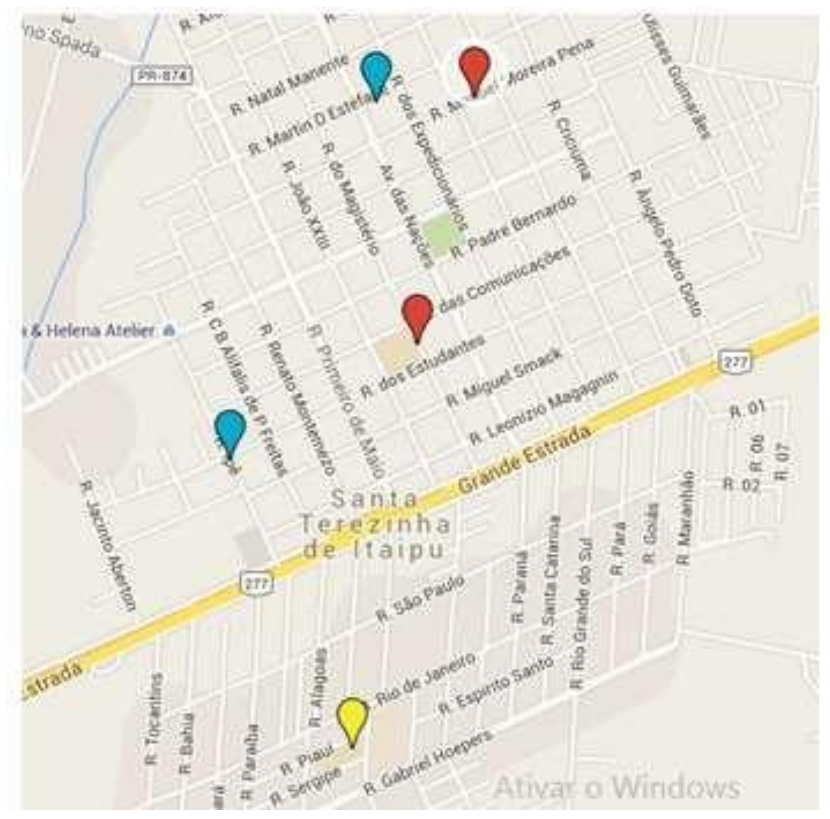

Figura 1. Localização das escolas municipais de Santa Terezinha do Itaipu/PR Fonte: Elaborada pelos autores a partir do Google Maps.

A partir da Figura 1, percebe-se que as escolas municipais estão próximas geograficamente e localizam-se no centro da cidade. Portanto, não há como afirmar que a localização influencia o custo das escolas.

\subsection{Análises de Desempenho}

Diante dos dados apresentados, procurou-se conhecer a realidade das unidades escolares destacadas de modo que fosse possível relacioná-las aos gastos públicos. A pesquisa se baseia no último Índice de Desenvolvimento da Educação Básica (IDEB) disponível, correspondente ao ano de 2013, que é disponibilizado pelo Instituto Nacional de Estudos e Pesquisas Educacionais Anísio Teixeira (INEP). 
Custos, Desempenho e Estrutura Educacional do Ensino Fundamental: Uma Análise em um Município do Interior do Paraná Lealis Vaz Meleiro Lopes, Caroline Nayara Marilac Flor, Marissa Yanara de Godoy Lima, Saulo Fabiano

Amâncio Vieira, Thais Accioly Baccaro

Tabela 4

Nota das escolas no IDEB

\begin{tabular}{|c|l|c|}
\hline & Nome da Escola & IDEB \\
\hline 1 & ALEXANDRE ZILLI NETTO E M EI EF & 6,8 \\
\hline 2 & CECILIA MEIRELES E M EI EF & 6,9 \\
\hline 3 & MONTEIRO LOBATO E M EI EF & 5,8 \\
\hline 4 & NOSSA SENHORA DO CARMO E M EI EF & 6,5 \\
\hline 5 & OLIMPIO SPRICIGO E M EF & 6,6 \\
\hline & Valores Médios & $\mathbf{6 , 5 2}$ \\
\hline
\end{tabular}

Nota. Fonte: Elaborada pelos autores.

A partir das informações expostas, nota-se que, em meio às 5 escolas municipais de Santa Terezinha do Itaipu, 4 apresentaram desempenho superior à meta do Plano de Educação, ou seja, $80 \%$ das unidades escolares analisadas obtiveram nota superior a 6 .

Para relacionar as variáveis NSE, custos e desempenho, elaborou-se o gráfico abaixo, que busca ilustrar as variáveis e as possíveis relações pertinentes às unidades escolares do município.

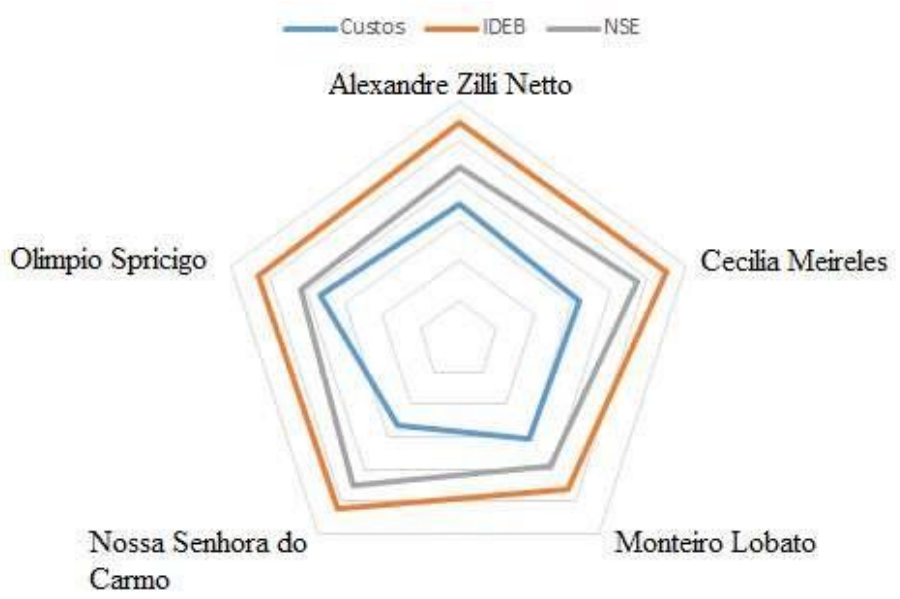

Figura 2. Relação entre IDEB, Custo/Aluno e NSE das escolas Fonte: Elaborada pelos autores. 
Custos, Desempenho e Estrutura Educacional do Ensino Fundamental: Uma Análise em um Município do Interior do Paraná Lealis Vaz Meleiro Lopes, Caroline Nayara Marilac Flor, Marissa Yanara de Godoy Lima, Saulo Fabiano Amâncio Vieira, Thais Accioly Baccaro

Mediante a figura apresentada, deve-se atentar ao fato de que a Escola Nossa Senhora do Carmo possui o menor custo por aluno da cidade (inferior ao valor gasto em nível nacional) e, ainda assim, conseguiu um desempenho próximo ao das demais escolas da cidade. Essa escola possui um Nível Socioeconômico alto, o que poderia explicar seu alto desempenho apesar dos baixos investimentos. Como já explanado no referencial teórico, desigualdade na distribuição da renda e deficiências no sistema educacional são problemas diretamente relacionados. Assim, ao se traçar uma política educacional, há de se evitar a posição simplista de que se pode resolver o problema da pobreza apenas abrindo escolas. Pobreza e ausência de escolarização são deficiências que somente poderão ser superadas se enfrentadas simultaneamente, cada uma em seu lugar próprio (Goldemberg, 1993).

É pertinente lembrar que o IDEB médio de Santa Terezinha do Itaipu pode ser considerado alto, visto que a meta estipulada pelo Plano de Educação é 6, e o desempenho apresentado pelas escolas do município é 6,52.

\section{CONSIDERAÇÕES FINAIS}

O estudo sobre custos na educação proporciona uma melhor visão do contexto atual, possibilitando uma série de melhorias na educação brasileira. A pergunta de pesquisa que orientou este estudo foi: As escolas de Santa Terezinha de Itaipu/PR que possuem o maior custo por aluno são também aquelas que ostentam o melhor desempenho no Índice de Desenvolvimento da Educação Básica (IDEB)? A resposta a essa questão é que as escolas com maior custo por aluno não foram aquelas que apresentaram melhor índice e, sim, aquelas nas quais as famílias tinham um nível socioeconômico mais elevado.

Santa Terezinha de Itaipu é uma cidade ainda em desenvolvimento, de pequeno porte, com cinco escolas mantidas pelo município. A cidade chama a atenção pelo IDEB alto, apresentando média de 6,2, enquanto o IDEB médio nacional é de 5,2. O custo/aluno é consideravelmente baixo, visto que a média das escolas municipais é de 
Custos, Desempenho e Estrutura Educacional do Ensino Fundamental: Uma Análise em um Município do Interior do Paraná Lealis Vaz Meleiro Lopes, Caroline Nayara Marilac Flor, Marissa Yanara de Godoy Lima, Saulo Fabiano Amâncio Vieira, Thais Accioly Baccaro

$R \$ 159,02$, e o custo/aluno médio nacional é de $R \$ 182,88$. Os anos iniciais do Ensino Fundamental compreendem o período escolar no qual a maior parte dos alunos se encontra.

A cidade direciona, em média, $87,09 \%$ do orçamento voltado à educação em custos pedagógicos que, dentre outros valores, incluem os salários dos professores, ou seja, o foco no docente pode ser um dos aspectos que influenciam no desempenho satisfatório das unidades escolares. O georreferenciamento não deixa claro se há uma relação entre localização da escola e custos, já que a maior parte delas fica relativamente próxima das outras.

Considerando que a escola com maior NSE apresenta o melhor desempenho no IDEB e a unidade com menor NSE é também a que apresenta menor nota no IDEB, acredita-se que o NSE possa influenciar diretamente no desempenho escolar, cabendo, portanto, ações provenientes do Estado que possam interferir nesse resultado.

Para pesquisas futuras, recomenda-se um estudo em maior profundidade da escola Nossa Senhora do Carmo que, dado seu baixo custo em parâmetro municipal e seu alto desempenho frente às demais, pode ser uma possível fornecedora de bons benchmarks.

Tratando-se de um estudo de caso em um município de pequeno porte, focado essencialmente em instituições públicas, o caso torna-se bastante específico e, possivelmente, não venha a refletir a realidade de outras unidades escolares de outros municípios. Ainda que não haja a possibilidade de generalização, o estudo é importante por propor distintas variáveis que podem influenciar no desempenho do Ensino Fundamental de outros municípios. A cidade de Santa Terezinha do Itaipu se destaca como um caso de sucesso no bom aproveitamento dos recursos, já que seu IDEB é maior do que a média nacional, e o custo por aluno é baixo. Sugerem-se estudos posteriores que se utilizem dessas mesmas variáveis para avaliar cidades de maior porte, além de estudos comparativos entre cidades com características similares. 
Custos, Desempenho e Estrutura Educacional do Ensino Fundamental: Uma Análise em um Município do Interior do Paraná Lealis Vaz Meleiro Lopes, Caroline Nayara Marilac Flor, Marissa Yanara de Godoy Lima, Saulo Fabiano Amâncio Vieira, Thais Accioly Baccaro

\section{REFERÊNCIAS}

Arelaro, L. R. G. (2005). O ensino fundamental no brasil: avanços, perplexidades e tendências. Educ. Soc., 26, n. 92, 1039-1066.

Brasil. (1988). Constituição. Constituição da República Federativa do Brasil. Centro Gráfico, 292.

Brasil. (2018). IBGE. Educação. Recuperado de: $<$ https://brasilemsintese.ibge.gov.br/educacao/taxa-de-escolarizacao-das-pessoasde-6-a-14-anos.html>.

Brasil. (2013). Lei 12.796, de 4 de abril de 2013. Altera, atualiza e consolida a legislação sobre as Diretrizes e Bases da Educação Nacional. Recuperado de: <http://www.planalto.gov.br/ccivil_03/leis/L9394.htm>.

Brasil. (2013). O que é IDEB. Recuperado de: <http://www.educacao.al.gov.br/indicadores/ideb/o-que-e-ideb>.

Brasil. Portal Qedu Academia. Glossário - Nível Socioeconômico. Recuperado de: $<$ http://academia.qedu.org.br/glossario/nivel-socioeconomico-nse>.

Carvalho, S. C., Borinelli, B., Suguihiro, V. L. T., \& Vieira, S. F. A. (2012). Indicadores de Gestão Pública Municipal, v. 1, 15-128.

De Oliveira, J. F., Libâneo, J. C., \& Toschi, M. S. (2017). Educação escolar: políticas, estrutura e organização. Cortez Editora.

Dourado, L. F. (2007). Políticas e gestão da educação básica no Brasil: limites e perspectivas. Recuperado de: <http://www.scielo.br/pdf/es/v28n100/a1428100.pdf>.

Fernandes, R. (2007). Índice de Desenvolvimento da Educação Básica (IDEB).

Goldemberg, J. (1993). O repensar da Educação no Brasil. Estudos Avançados. Recuperado de: <http://www.scielo.br/scielo.php?script=sci_arttext\&pid=S010340141993000200004>.

Jackson, A., \& Lapsley, I. (2003). The diffusion of accounting practices in the new "managerial" public sector. International Journal of Public Sector Management, 16 (5), 359-372.

Martins, E. (2009). Contabilidade de custos. Eliseu. (9a ed). São Paulo: Atlas. 
Custos, Desempenho e Estrutura Educacional do Ensino Fundamental: Uma Análise em um Município do Interior do Paraná Lealis Vaz Meleiro Lopes, Caroline Nayara Marilac Flor, Marissa Yanara de Godoy Lima, Saulo Fabiano Amâncio Vieira, Thais Accioly Baccaro

Prefeitura de Santa Terezinha de Itaipu. (2016). Portal de Santa Terezinha de Itaipu. Recuperado de: <http://www.stitaipu.pr.gov.br>.

Portal População. Net. População. (2016). Recuperado de: $<$ http://populacao.net.br/populacao-santa-terezinha-de-itaipu_pr.html>.

Portal Cidades do Meu Brasil. (2016). Santa Terezinha de Itaipu. Recuperado de: <http://www.cidadesdomeubrasil.com.br/PR/santa_terezinha_de_itaipu>.

Sistema Integrado de Custos Municipais. (2015). Apresentação 2015. Recuperado de: <https://sicm.dc.uel.br/home>.

Soares, J. F., \& Pereira, X. F. (2013). Pressupostos educacionais e estatísticos do Ideb - Educação \& Sociedade. 2013 (Julho-Setembro). Recuperado de: <http://www.scielo.br/scielo.php?script=sci_arttext\&pid=S010173302013000300013 $>$.

Vendramin Junior, V. (2015). Gestão de custos para qualidade e eficiência dos gastos públicos: mapeamento da percepção dos gestores públicos e contadores da Região Metropolitana de Campinas (RMC). (Dissertação de Mestrado). Fundação Escola de Comércio Álvares Penteado - FECAP. Recuperado de: $<$ http://tede.fecap.br:8080/jspui/bitstream/tede/669/5/Vilson_Vendramin_Junior.pdf>.

Data de Submissão: 17/01/2017

Data de Aceite: 21/02/2018 